\title{
Potent antitumoral activity of TRAIL through generation of tumor-targeted single-chain fusion proteins
}

\author{
B Schneider ${ }^{1}$, S Münkel ${ }^{1}$, A Krippner-Heidenreich ${ }^{2}$, I Grunwald ${ }^{3}$, WS Wels ${ }^{4}$, H Wajant $^{5}$, K Pfizenmaier ${ }^{\star, 1}$ and J Gerspach ${ }^{1}$
}

In an attempt to improve TRAIL's (tumor necrosis factor-related apoptosis-inducing ligand) tumor selective activity a variant was designed, in which the three TRAIL protomers are expressed as a single polypeptide chain (scTRAIL). By genetic fusion with a single-chain antibody fragment (scFv) recognizing the extracellular domain of ErbB2, we further equipped scTRAIL with tumortargeting properties. We studied tumor targeting and apoptosis induction of scFv-scTRAIL in comparison with non-targeted scTRAIL. Importantly, the tumor antigen-targeted scTRAIL fusion protein showed higher apoptotic activity in vitro, with a predominant action by TRAIL-R2 signaling. Pharmacokinetic studies revealed increased plasma half-life of the targeted scTRAIL fusion protein compared with ScTRAIL. In vivo studies in a mouse tumor model with xenotransplanted Colo205 cells confirmed greater response to the ErbB2-specific scTRAIL fusion protein compared with non-targeted scTRAIL both under local and systemic application regimen. Together, in vitro and in vivo data give proof of concept of higher therapeutic activity of tumor-targeted scFv-scTRAIL molecules. Further, we envisage that through targeting of scTRAIL, potential side effects should be minimized. We propose that scFv-mediated tumor targeting of single-chain TRAIL represents a promising strategy to improve TRAIL's antitumoral action and to minimize potential unwanted actions on normal tissues.

Cell Death and Disease (2010) 1, e68; doi:10.1038/cddis.2010.45; published online 26 August 2010

Subject Category: Cancer

Although for many malignancies traditional cytotoxic chemotherapy is still the treatment of choice, targeted therapies gain increasing importance because they are expected to exert higher tumor-specific activity and less dose-limiting side effects. The anti-CD20 antibody Rituximab and the Bcr-Abl-selective kinase inhibitor Gleevec, respectively, are two vivid examples for the success of targeted therapy (for review, see Gerber ${ }^{1}$ ). Targeting the apoptotic machinery of malignant cells has become an attractive concept, beginning with tumor necrosis factor (TNF) and its now established clinical use for locoregional treatment of limb metastases of soft tissue sarcoma and melanoma ${ }^{2}$ and followed by the current exploitation of other proapoptotic members of the TNF ligand family - CD95L and TNF-related apoptosisinducing ligand (TRAIL) - being presently at various stages of preclinical and clinical development (for review, see Call et al., ${ }^{3}$ Gerspach et al. ${ }^{4}$ and Newsom-Davis et al. ${ }^{5}$ ). TRAIL, similar to TNF a type II transmembrane protein, is expressed on various immune effector cells and appears critically involved in antitumor responses in the body. ${ }^{6}$ It is probably the best candidate of a death ligand for systemic application because preclinical studies revealed that, in contrast to TNF and CD95 agonists, the application of trimeric, soluble TRAIL is well tolerated. Concomitantly, it demonstrated potent antitumor activity in tumor models based on tumor cell lines and primary tumor samples. ${ }^{7-10}$ Although first clinical trials in various advanced cancers are promising, as stable disease has been reported in many cases, TRAIL monotherapy most likely will not result in a sufficient overall therapeutic activity. ${ }^{11}$ Concurrently, this faces the potential problem that under combined treatment options normal cells might also be sensitized to TRAIL-induced apoptosis (reviewed by Falschlehner et al. ${ }^{12}$ ). Furthermore, we have recently shown that TRAIL-R1 is activated by the soluble as well as the membrane-bound form of the ligand, whereas TRAIL-R2 is preferentially activated by membrane TRAIL or oligomerized soluble trimeric TRAIL. ${ }^{13,14}$ As agonistic TRAIL-R2specific antibodies likewise display potent antitumor activity (reviewed by Johnstone et al. ${ }^{15}$ ), it is conceivable that homotrimeric TRAIL could be improved in its activity provided efficient TRAIL-R2 signaling is ensured through genetic modifications or other means. On the other hand, the spontaneous

\footnotetext{
${ }^{1}$ Institute of Cell Biology and Immunology, University of Stuttgart, Allmandring 31, Stuttgart 70569, Germany; ${ }^{2}$ Institute of Cellular Medicine, Newcastle University, 4th Floor Catherine Cookson Building, Framlington Place, Newcastle upon Tyne NE2 4HH, UK; ${ }^{3}$ Fraunhofer Institute for Manufacturing Technology and Applied Materials Research IFAM, Bonding Technology and Surfaces, Adhesives and Chemistry of Polymers, Wiener Straße 12, Bremen 28359, Germany; ${ }^{4}$ Chemotherapeutisches Forschungsinstitut Georg-Speyer-Haus, Paul-Ehrlich-Straße 42-44, Frankfurt 60596, Germany and ${ }^{5}$ Division of Molecular Internal Medicine, Department of Internal Medicine II, University Hospital of Würzburg, Röntgenring 11, Würzburg 97070, Germany

${ }^{*}$ Corresponding author: K Pfizenmaier, Institute of Cell Biology and Immunology, University of Stuttgart, Allmandring 31, Stuttgart 70569, Germany.

Tel: + 49711685 66986; Fax: + 49711685 67484; E-mail: klaus.pfizenmaier@izi.uni-stuttgart.de

Keywords: tumor targeting; single-chain TRAIL; scFv-TRAIL fusion proteins; apoptosis; ErbB2

Abbreviations: i.v., intravenous(ly); TNF, tumor necrosis factor; TRAIL, TNF-related apoptosis-inducing ligand; TRAILR1/2, TRAIL receptor 1/2; scFv, single-chain Fv fragment; scTRAIL, single-chain TRAIL; s.c., subcutaneous(ly)

Received 19.4.10; revised 02.7.10; accepted 14.7.10; Edited by R De Maria
} 
aggregation of some recombinant TRAIL preparations, resembling secondarily crosslinked TRAIL, has been associated with unwanted actions on normal tissues and thus curtail their use as tumor-selective apoptosis inducers (reviewed by Koschny et al. ${ }^{16}$ ). Thus, TRAIL variants mimicking the full bioactivity of membrane TRAIL could present powerful cancer therapeutics provided that a tumorrestricted action can be achieved. A further apparent limitation of the currently clinically investigated recombinant TRAIL reagents is their rather short in vivo half-life. Therefore, an improvement of pharmacokinetic properties might also lead to increased therapeutic action. A potential solution to increase specific activity and bioavailability is the combination of TRAIL function with tumor targeting. Thus, we generated new TRAIL fusion proteins containing an antibody fragment (single-chain Fv fragment (scFv)) for targeting TRAIL to the tumor to enrich the therapeutic at the tumor site and to enhance its specific bioactivity. ErbB2, similar to EGFR/ErbB1, ErbB3 and ErbB4 belonging to the ErbB receptor tyrosine kinase family, represents a clinically relevant target antigen. It is highly expressed on a variety of human solid tumors (reviewed by Holbro and Hynes ${ }^{17}$ ) and there is already one humanized monoclonal antibody $(\mathrm{mAb})$ directed against the extracellular domain of ErbB2 in clinical use (Trastuzumab) and approved for treatment of breast carcinoma. Several other mAbs are in different stages of clinical evaluation (reviewed by Baselga and Swain ${ }^{18}$ ). Although the clinical data provide clear evidence that ErbB2 is a relevant target, they also show that the therapeutic effect of the current antibody reagents is rather limited. ${ }^{19}$ This justifies the search for additional ErbB2-targeted strategies.

Recently, a single-chain TNF molecule, consisting of three TNF monomers fused by short peptide linkers, was described to exert enhanced stability and antitumoral activity. ${ }^{20} \mathrm{We}$ have designed an analogous single polypeptide chain TRAIL variant (scTRAIL), which was used to construct a scFv-scTRAIL fusion protein for tumor targeting. We have compared the bioactivities of this fusion protein with nontargeted ScTRAIL in in vitro and in vivo tumor models. We show here that the tumor antigen-targeted ScTRAIL fusion protein showed higher apoptotic activity to ScTRAIL in vitro, with a predominant action by TRAIL-R2 signaling on Colo205 colon carcinoma cells. In vivo studies in a mouse xenograft tumor model confirmed significantly higher response to the tumor-targeted ErbB2-specific scTRAIL fusion protein.

\section{Results}

Construction and production of TRAIL fusion proteins. To generate a single-chain TRAIL molecule, we followed the design principle described for scTNF ${ }^{20}$ and covalently fused three TRAIL monomers, each consisting of the extracellular domain of TRAIL (aa 95-281) through two peptide linkers comprising four repeats of the sequence GGGS (Figure 1a). To facilitate purification and analysis an N-terminal FLAG tag was added. Furthermore, by additional N-terminal fusion of a single-chain antibody fragment (scFv) recognizing the FRP5 epitope within the extracellular domain of ErbB2, ${ }^{21}$ we generated an ErbB2-specific scTRAIL fusion protein (Figure 1a).
Both scTRAIL and ScFv-scTRAIL were purified by affinity chromatography on monoclonal M2 anti-FLAG agarose from the supernatant of stably transfected HEK293 cells with yields of about $1 \mathrm{mg}$ protein per liter cell culture supernatant. Immunoblot analysis and SDS-PAGE (Figure 1b) of the purified protein showed single protein bands with a molecular mass of $\sim 70 \mathrm{kDa}$ for scTRAIL and $\sim 100 \mathrm{kDa}$ for scFvscTRAIL matching the expected calculated molecular masses of 71 and $98 \mathrm{kDa}$, respectively. Gel filtration analysis indicated a monomeric organization of both ScTRAIL variants, corresponding, with respect to the TRAIL part, to a noncovalently assembled trimer of a conventional recombinant TRAIL molecule (Figure 1c). The molecular masses deduced from SEC were slightly lower compared with that derived from SDS-PAGE (Figure 1b), which did not result from degradation as verified by immunoblot analysis of collected fractions (Figure 1c). In the scTRAIL preparation, the minor fraction eluting in SEC at apparent higher molecular weight, thus potentially comprising aggregated complexes, did not display disproportionately high bioactivity, as revealed from comparison of cytotoxic activity of fraction 2 and 15, taking relative protein amounts into consideration (data not shown).

Target antigen-specific binding of scFv-scTRAIL. To analyze specific binding of scFv-scTRAIL to ErbB2-positive cells, flow cytometry analysis was performed. ErbB2 expression analysis confirmed a median versus low expression level for Colo205 colon carcinoma and HT1080 fibrosarcoma cells, respectively (Figure 2a), compared with SKBR3 cells that are well-known to highly express the ErbB2 protein (data not shown). ${ }^{22}$ Incubation of Colo205 and HT1080 cells with the fusion protein revealed a specific, binding to ErbB2positive cells (Figure $2 a$ and $b$ ) compared with incubation with the non-targeted ScTRAIL, which resulted only in a weak fluorescence signal, either by detection with anti-TRAIL (Figure 2a) or with anti-FLAG antibodies (data not shown). As binding of homotrimeric FLAG-tagged TRAIL showed similar weak signals (data not shown), this could reflect a low level of TRAIL receptor (TRAILR) expression on the investigated target cells and/or insufficient sensitivity of FACS analyses. ErbB2-specific binding was confirmed by using TRAILR1- and R2-Fc fusion proteins for detection of ScFv-scTRAIL binding to target cells (Figure $2 \mathrm{~b}$ ) and competition with anti-ErbB2 antibodies (FRP5). This led to a near complete inhibition of ScFv-scTRAIL binding to the ErbB2-positive cell line (Figure 2b).

Target antigen-restricted induction of cell death by scFv-scTRAIL. To address the bioactivity of scFvScTRAIL in vitro, we first analyzed induction of cell death on ErbB2-negative SKW6.4 cells, which are known to be TRAIL-R1 and TRAIL-R2 positive ${ }^{23,24}$ (Figure 3a). Although SCTRAIL and ScFv-scTRAIL did not show cytotoxic activity up to $8 \mathrm{nM}$, respectively, in the presence of the crosslinking anti-FLAG antibody M2 comparable bioactivities of both fusion proteins were observed (Figure 3a). In contrast, incubation of ErbB2-positive Colo205 and HT1080 cells with ScTRAIL or ScFv-scTRAIL revealed an approximately three- to fivefold higher bioactivity of the ErbB2-specific scFv-scTRAIL compared with the non-targeted scTRAIL 
a
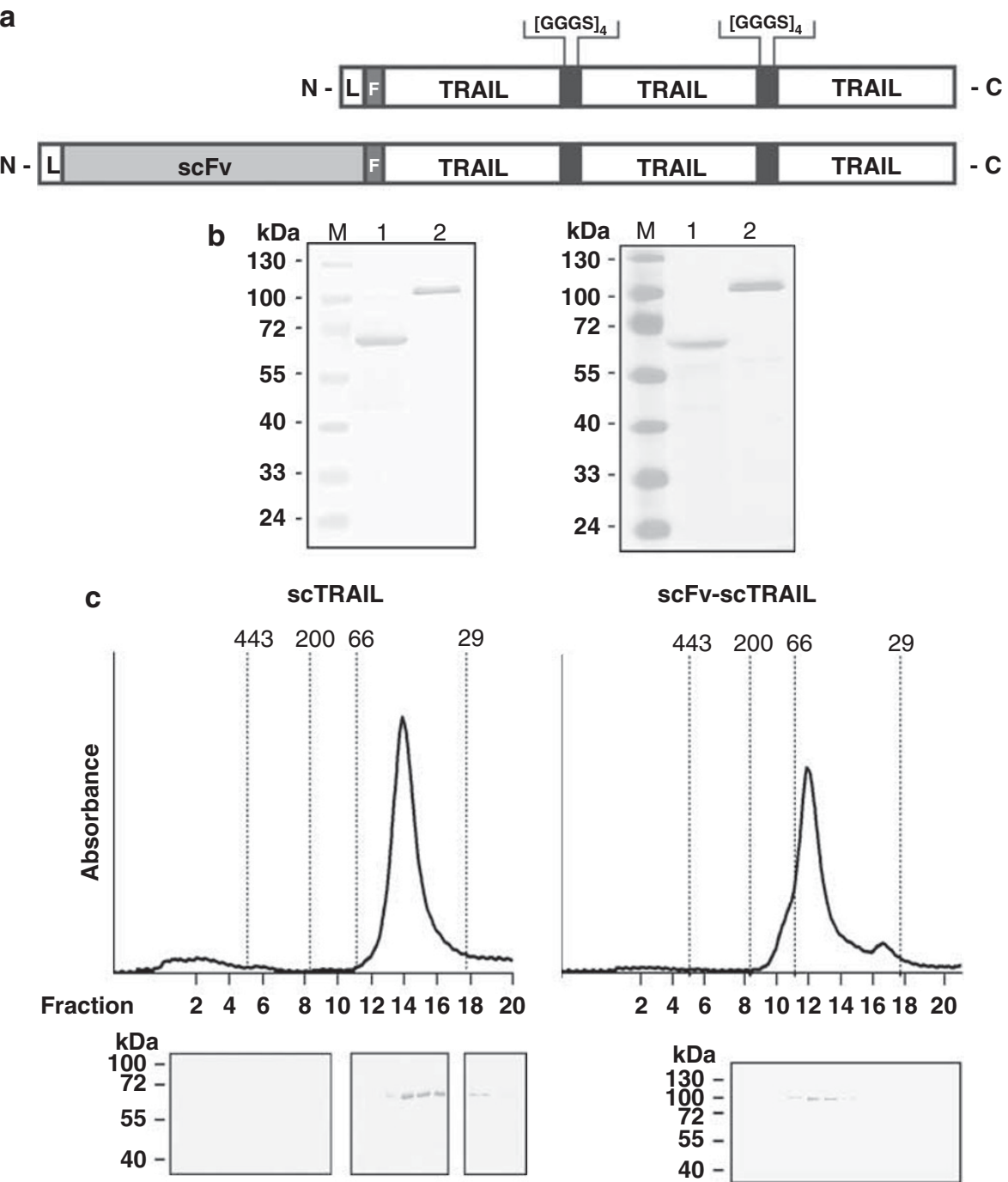

Figure 1 Biochemical analysis of scTRAIL and scFv-scTRAIL. (a) Scheme of the scTRAIL fusion proteins used in this study. L, leader peptide; scFv, human ErbB2specific single-chain antibody fragment; F, FLAG tag; TRAIL, human TRAIL (aa 95-281). (b) Purified scTRAIL (lane 1) and scFv-scTRAIL (lane 2) were analyzed by SDS-PAGE (10\%, reducing conditions) followed by immunoblot analysis (left, $250 \mathrm{ng}$ protein/lane) or silver staining (right, $1 \mu \mathrm{g}$ protein/lane). For immunoblotting, the monoclonal anti-FLAG M2 antibody and an alkaline phosphatase-conjugated secondary antibody was used. (c) The TRAIL variants were separated by gel filtration on a BioSuite250 column. Dotted lines indicate the retention time of the molecular weight standards apoferritin (443 kDa), $\beta$-amylase (200 kDa), bovine serum albumin ( $66 \mathrm{kDa})$ and carbonic anhydrase $(29 \mathrm{kDa})$. Collected fractions were analyzed by immunoblotting as in $\mathbf{b}$. For scTRAlL fractions 1-9 (every second fraction; first part), fractions 11-16 (second part) and fractions 19-26 (every second fraction; third part) were immunoblotted. For scFv-scTRAIL fractions 8-24 were immunoblotted

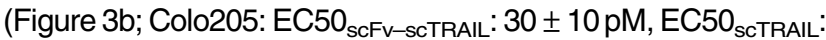

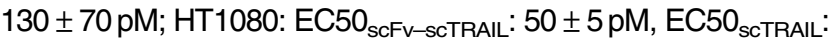
$140 \pm 10 \mathrm{pM})$. Furthermore, bioactivity of the targeted scFv-scTRAIL compared well with that of a commercially available high activity TRAIL, the so-called 'Killer-TRAIL', with about twofold higher (Colo205) or at least comparable (HT1080) activity of the ScFv-scTRAIL reagent (Figure 3c). Furthermore, on target-negative SKW6.4 cells, the secondarily crosslinked SCTRAIL or ScFv-scTRAIL displayed comparable cytotoxic activity to 'Killer-TRAIL' (data not shown). From blocking studies using anti-ErbB2 antibodies (FRP5, Figure 3d) or recombinant ErbB2 peptides comprising the epitope of the erbB2-specific scFv ${ }^{21}$ (Figure $3 e$ ), it became evident that the increased bioactivity depended on ErbB2 target binding. Preincubation with FRP5 or ErbB2 peptides before addition of the ErbB2-specific ScFv-scTRAIL fusion protein restored cell viability of Colo205 and HT1080 cells to a significant extent (Figure 3d and e). Furthermore, as for both tested cell lines TRAILinduced cell death is primarily mediated by TRAIL-R2, ${ }^{13,25}$ the higher bioactivity of the ScFv-scTRAIL on ErbB2-positive cells pointed to a membrane TRAIL-mimetic activity upon specific cell surface immobilization of the fusion protein (see below). Furthermore, inhibition of TRAIL bioactivity of the scFv-scTRAIL by the use of a TRAIL-neutralizing antibody led to complete restoration of cell viability of Colo205 and HT1080 cells (Figure 3f). Thus, the ErbB2-specific scFv domain per se did not directly contribute to apoptosis induction. 


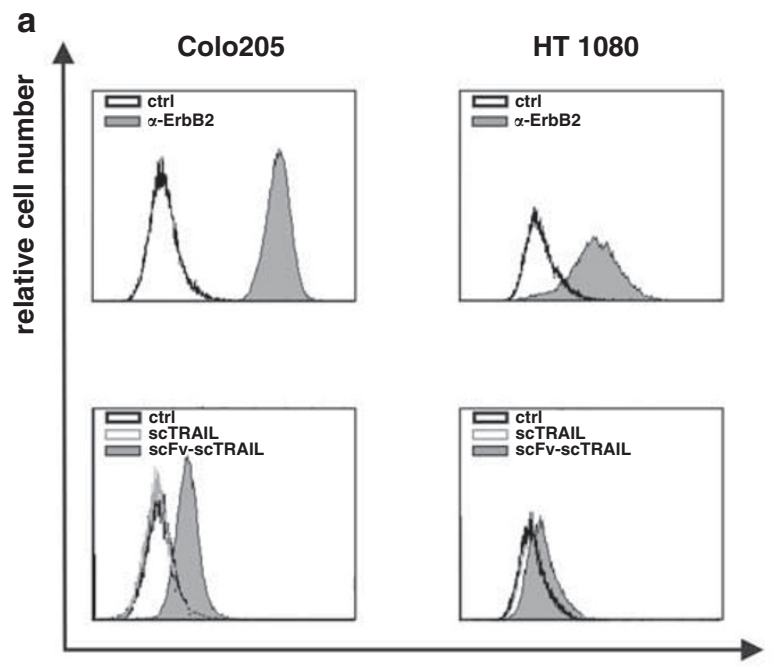

log fluorescence intensity
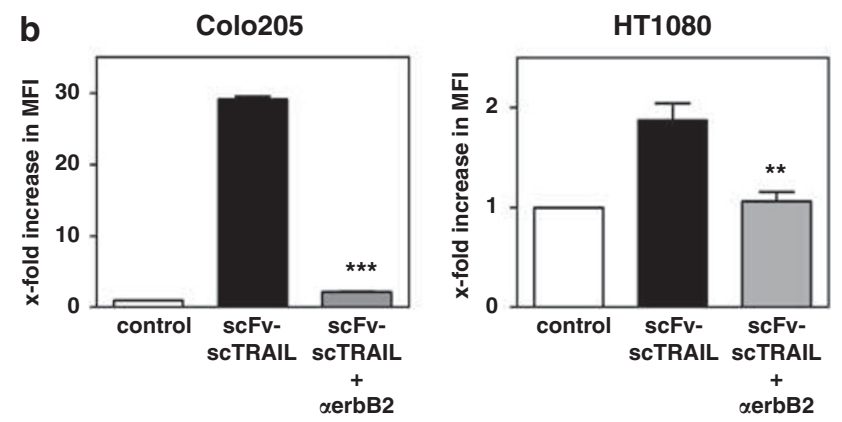

Figure 2 Target antigen-specific binding of scFv-scTRAIL. (a) ErbB2 expression of Colo205 and HT1080 cells: $5 \times 10^{5}$ cells were incubated with anti-ErbB2 (gray filled) or isotype control antibody (black lines) followed by FITC-conjugated anti-mouse antibody and fluorescence intensity was measured by FACS analysis. Binding of scFv-scTRAIL to ErbB2-positive Colo205 and HT1080 cells compared with non-targeted SCTRAIL: $5 \times 10^{5}$ cells were incubated with $50 \mathrm{nM}$ scTRAIL (gray lines) or $50 \mathrm{nM} \mathrm{scFv-scTRAIL} \mathrm{(gray} \mathrm{filled)} \mathrm{and,} \mathrm{after} \mathrm{repeated}$ washes, bound proteins were detected using anti-human TRAIL mAb and FITC-labeled anti-mouse IgG. As control, cells treated with anti-humanTRAIL mAb and FITC-labeled anti-mouse IgG were used (black lines). (b) Binding competition of scFv-scTRAIL to ErbB2-positive Colo205 cells by ErbB2-specific antibodies: $5 \times 10^{5}$ Colo205 or HT1080 cells were preincubated with FRP5 or mouse IgG1 $(10 \mu \mathrm{g} / \mathrm{ml}$, respectively) before addition of scFv-scTRAIL $(50 \mathrm{nM})$. Detection was carried out by TRAILR-Fc fusion proteins and FITC-labeled anti-human IgG, Fc-specific antibodies. Shown are X-fold increased mean fluorescence intensities (MFI) compared with the control (FITC-labeled anti-human FC) from three independent experiments (mean \pm S.D.). Statistical analysis was carried out with unpaired $t$-test, two-tailed test, ${ }^{\star \star} P=0.0021,{ }^{\star \star *} P<0.0001$

TRAIL-R2-mediated apoptosis induction of scFvScTRAIL. In absence of the ErbB2 antigen, crosslinking of scFv-scTRAIL by its internal FLAG tag with the FLAG-specific mAb M2 was necessary to induce cell death in SKW (Figure 3a) and in Jurkat cells (Figure 4a). Thus, similar to homotrimeric TRAIL, secondary crosslinking conferred TRAILR2-stimulating capacity, confirming the nonaggregated state of the single-chain fusion protein with a TRAIL domain resembling a trimeric soluble TRAIL. The ErbB2-positive Colo205 and HT1080 cells express both, TRAIL-R1 and -R2. To define through which of the two TRAIL death receptors the
scFv-scTRAIL molecule signals apoptosis, neutralization experiments with antagonistic, TRAIL-R2-specific monovalent antibody fragments (Fab fragments) were performed. Both ErbB2-positive cell lines showed dominant TRAIL-R2mediated cell death at low concentrations of ScFv-scTRAIL (Figure 4b), demonstrating that cell surface immobilized scFvscTRAIL exerted membrane TRAIL-like activity, similar to what has been previously described for conventional scFv-TRAIL fusion proteins. ${ }^{13,26,27}$ The concentration of anti-TRAIL-R2 Fab fragments used in these experiments was sufficient to almost completely block cell death of Jurkat cells induced by secondary crosslinked, homotrimeric FLAG-tagged TRAIL (Figure 4b). In addition, cell surface antigen-bound scFvSCTRAIL also activated the murine TRAILR, which is, similarly to human TRAIL-R2, not or hardly activated by soluble TRAIL. This was shown by mixed cultures using equal numbers of Colo205 cells and L929 mouse fibroblasts (Figure 4c). The latter do not express human ErbB2 and thus, in contrast to Colo205 cells, are not able to immobilize the scFv-scTRAIL by the ErbB2-targeting domain. Thus, if scFv-scTRAIL is able to induce cell death in L929 cells by mimicking membrane TRAIL after binding to the ErbB2 receptor on Colo205 cells, more than $50 \%$ of the cells are expected to die. Indeed, incubation with ScFv-scTRAIL induced cell death in over $70 \%$ cells of the co-culture, whereas equal molar amounts of ScTRAIL induced cell death in only about $30 \%$ of the mixed cells (Figure $4 \mathrm{c}$ ). In monocultures, the same concentration of scFv-scTRAIL and scTRAIL induced cell death in about $90 \%$ of Colo205 cells, respectively, but not in L929 cells, confirming that, in the soluble form, both proteins are non-toxic to the latter (data not shown). Furthermore, due to the lack of human ErbB2, the L929 cells might also be considered as antigen-negative bystander cells, potentially occurring in a tumor tissue. Thus, scFv-scTRAIL bound to antigen is expected to elicit an antitumoral effect also on neighboring antigen-negative cells within the tumor environment.

Serum half-life of ScFv-scTRAIL. To evaluate the pharmacokinetic characteristics of ScFv-scTRAIL in comparison with ScTRAIL, serum samples were collected after a single dose intravenous (i.v.) injection of $400 \mathrm{pmol}$ into Balb/c mice. The relative serum concentrations plotted over time fitted well to a two-phase exponential decay regression curve in both cases. Within $20 \mathrm{~min}$ post-injection, both TRAIL variants displayed similar kinetics with a serum half-life of $\sim 35 \mathrm{~min}$ $\left(t_{\frac{1}{2}} \alpha\right)$, respectively. At later time points, a slight but significant difference in bioavailability was detected with half-lifes $\left(t_{\frac{1}{2}} \beta\right)$ of 160 and $124 \mathrm{~min}$ and AUCs of 11191 and 8125 for scFvSCTRAIL and ScTRAIL, respectively (Figure 5a). Thus, the ErbB2-specific fusion protein was more efficiently retained in the bloodstream, which might positively affect therapeutic activity.

Tumoricidal effects on human colon carcinomas in nude mice. To investigate whether the improved target antigenrestricted tumoricidal activity of ScFv-scTRAIL observed in vitro is effective in vivo, in a first experiment a local treatment strategy was applied. NMRI nu/nu mice were injected subcutaneously (s.c.) with Colo205 cells. Treatment commenced 8 days later when vascularized tumor could be 

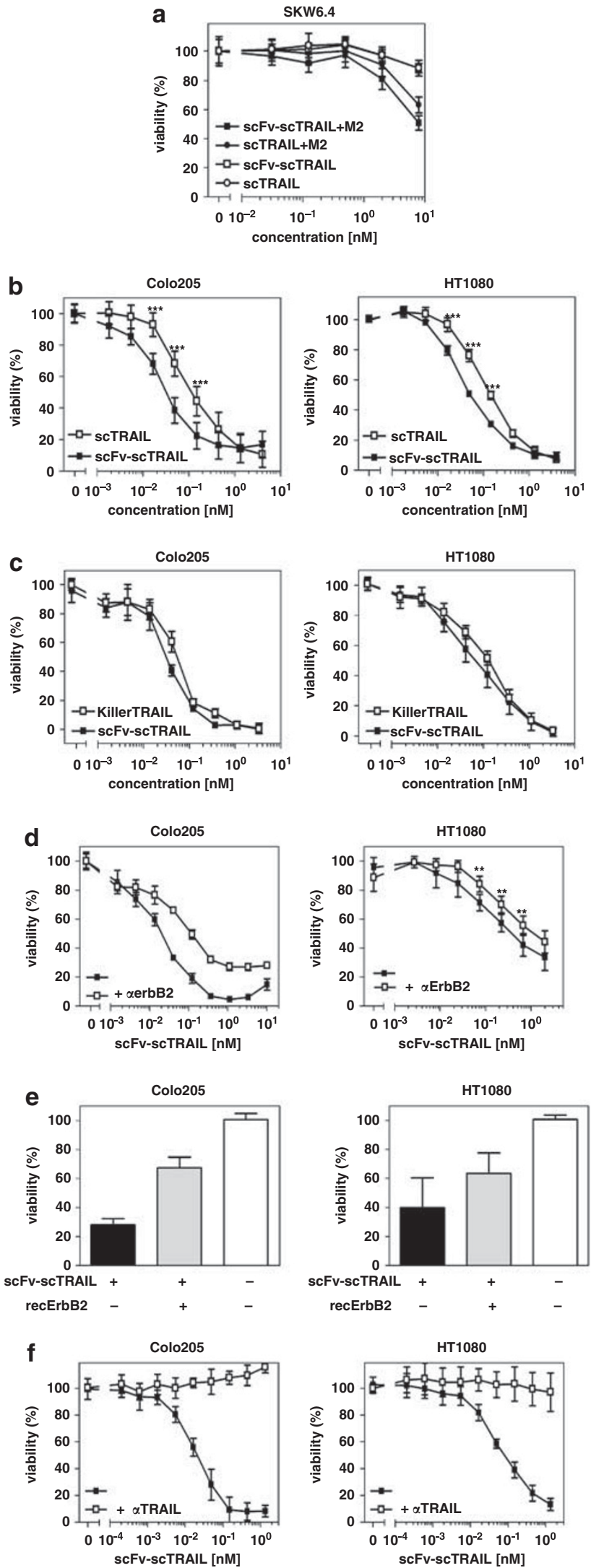

clearly discerned. Regimen was 4 daily s.c. injections of $1 \mathrm{nmol}$ of ScTRAIL and ScFv-scTRAIL, respectively, in an area close to the established tumors. Tumor growth was monitored for 22 days. With this treatment protocol, we observed significant tumor regression in the ScFv-scTRAILtreated group compared not only with the control group (days $1-19$; e.g., day $11,{ }^{\star \star} P=0.003$ ) but also with the nontargeted ScTRAIL-treated group (days 1-11; e.g., day 11, ${ }^{\star} P=0.04$, Figure $5 \mathrm{~b}$ ). These data are in accordance with one pilot experiment also showing significantly reduced tumor growth up to 11 days after ScFv-scTRAIL treatment started $\left({ }^{\star *} P<0.003\right.$, data not shown). These data prompted studies with a systemic treatment protocol to assess the feasibility of such fusion proteins for potential clinical use. Again treatment was initiated when a solid, vascularized tumor had been established. Mice received 8 daily i.v. injections of $1 \mathrm{nmol}$ of the TRAIL fusion proteins or control injections. All treatments were well tolerated by the animals, and we observed no weight loss or other obvious signs of systemic toxicity. Tumor size in control mice increased progressively over the study period (Figure $5 \mathrm{c}$ ). Similar to the peritumoral treatment, systemic application of the two TRAIL reagents resulted in clear antitumoral activity during treatment. We observed significantly delayed tumor growth on ScTRAIL treatment compared with control groups (days 1-19), and an even stronger effect for the tumor-targeted ScFv-scTRAIL fusion protein (days 1-19). Remarkably, during ScFvScTRAIL treatment a clear reduction in tumor size was recorded, which resulted in a significantly enhanced antitumoral effect compared with non-targeted scTRAIL (days 1-19). Although tumors of both treatment groups began to re-grow shortly after discontinuation of treatment, these differences remained significant during the complete observation period (e.g., day 19 (Figure 5c)).

\section{Discussion}

We here show that through genetic engineering and targeting, TRAIL's antitumoral activity can be improved. TRAIL is considered as a tumor-selective agent, as cells from normal

Figure 3 Target antigen-dependent bioactivity of ScFv-scTRAIL. (a) Bioactivity on target antigen-negative cells. $2 \times 10^{4} \mathrm{SKW6.4}$ cells/well were seeded in 96-well plates. The next day, cells were treated with serial dilutions of the fusion proteins in the presence or absence of anti-FLAG M2 antibody $(2 \mu \mathrm{g} / \mathrm{ml})$. (b) Bioactivity of scTRAIL versus scFv-scTRAIL. $5 \times 10^{4}$ Colo205 or $2 \times 10^{4}$ HT1080 cells/well were seeded in 96-well plates. The following day, cells were treated with $2.5 \mu \mathrm{g} / \mathrm{ml}$ cycloheximide and challenged in duplicates with increasing concentrations of scTRAIL and scFv-scTRAIL, respectively. After over night incubation, cell viability was determined by MTT assay or KV staining. Results from three to five independent experiments are shown (mean \pm S.D.). (c) Bioactivity of scFvScTRAIL compared to 'KillerTRAIL': Same assay as in b but incubation with serial dilutions of scFv-scTRAIL or 'KillerTRAIL'. (d, e) Target antigen-dependent apoptosis induction of scFv-scTRAIL: Same assay as in b with scFv-scTRAIL titrated in the presence or absence of anti-ErbB2 (FRP5, $5 \mu \mathrm{g} / \mathrm{ml}$ ) (d) or with scFvscTRAIL $(0.05 \mathrm{nM})$ in the presence or absence of recErbB2 $(10 \mu \mathrm{g} / \mathrm{ml})(\mathrm{e})$. (f) Contribution of the scFv domain in the scFv-scTRAIL to apoptosis induction. Same assay as in $\mathbf{b}$ with scFv-scTRAIL titrated in the presence or absence of neutralizing anti-TRAIL antibodies (2E5, $1 \mu \mathrm{g} / \mathrm{ml})$. (a, c-f) Results from three independent experiments are shown (mean \pm S.D.). (b, d) Statistical analysis was carried out with unpaired $t$-test, two-tailed test, ${ }^{* \star} 0.0026<P>0.0098,{ }^{* \star *} P<0.0001$ 

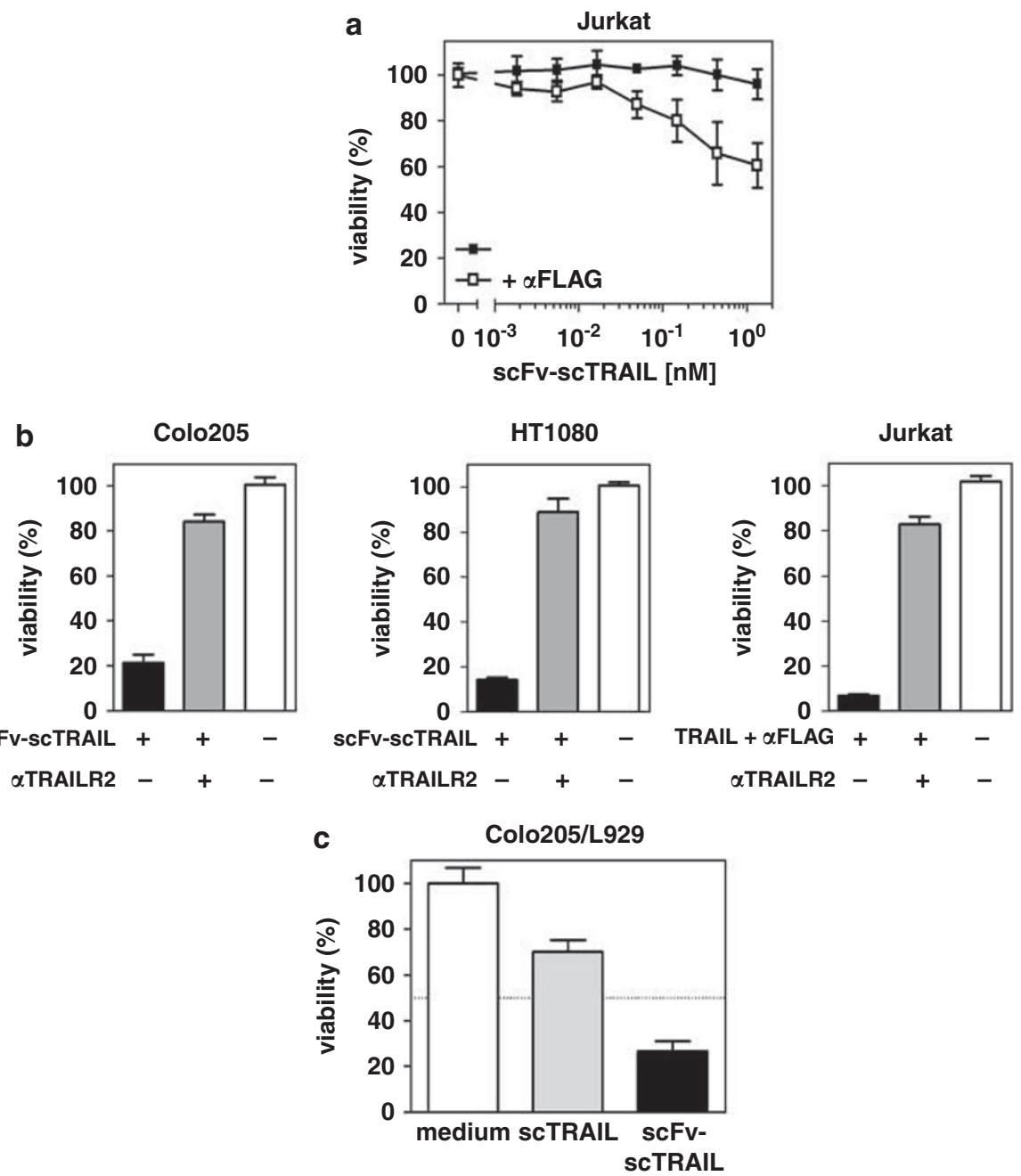

Figure 4 TRAIL-R2-mediated apoptosis induction of scFv-scTRAIL. (a) $1 \times 10^{5} \mathrm{Jurkat}$ cells/well were seeded in 96-well plates. The following day, cells were challenged with the indicated concentration of scFv-scTRAIL in the absence (open symbols) or presence (filled symbols) of $1 \mu \mathrm{g} / \mathrm{ml}$ of the crosslinking anti-FLAG mAb M2. After additional $16 \mathrm{~h}$, cell viability was determined by MTT assay. (b) Cells $\left(5 \times 10^{4}\right.$ Colo205, $2 \times 10^{4} \mathrm{HT} 1080,1 \times 10^{5}$ Jurkat cells/well) were seeded in 96 -well plates. The next day, cells were treated with $2.5 \mu \mathrm{g} / \mathrm{ml}$ cycloheximide (HT1080, Colo205) and challenged with scFv-scTRAIL (1.35 nM) (HT1080, Colo205) or $2 \mathrm{nM}$ TRAIL $+1 \mu \mathrm{g} / \mathrm{ml}$ anti-FLAG mAb M2 (Jurkat) in the presence or absence of TRAIL-R2 Fab $(50 \mu \mathrm{g} / \mathrm{ml})$. After additional $16 \mathrm{~h}$, cell viability was determined by MTT assay or KV staining. (c) $1 \times 10^{4}$ Colo205 and $1 \times 10^{4} \mathrm{~L} 929 \mathrm{cells} /$ well were seeded and co-cultured in 96-well plates. The next day, cells were treated with $2.5 \mu \mathrm{g} / \mathrm{ml}$ cycloheximide and challenged with $1 \mathrm{nM}$ scTRAlL or scFv-scTRAIL. After additional $16 \mathrm{~h}$, cell viability was determined by MTT assay. (a-c) Results from three independent experiments are shown (mean \pm S.D.)

tissue appear largely resistant toward apoptosis induction by trimeric TRAIL derivatives, ${ }^{8}$ and clinical studies so far revealed that homotrimeric TRAIL is well tolerated. ${ }^{28,29}$ However, in contrast to many investigated tumor cell lines, diverse primary cancer cells are inherently resistant to TRAIL-mediated apoptosis (reviewed by Newsom-Davis et al. ${ }^{5}$. Moreover, it has been demonstrated that TRAIL may under certain conditions even promote survival, proliferation, migration and invasion in TRAIL-resistant tumor cells. ${ }^{30,31}$ Thus, combination therapy with other drugs will be required, for example, for sensitization of apoptotic pathways or suppression of potentially protumoral TRAIL effects, to achieve major and lasting tumor remissions. In this context, recent data appear of relevance showing that the resistance of nontransformed cells toward the apoptotic action of TRAIL may vanish, at least in some tissues such as the liver, when these are subjected to various stress conditions, such as infection and chemotherapeutics. ${ }^{12,32-34}$ Recently, it has also been shown that TRAIL-R2-mediated apoptosis might substantially contribute to chronic cholestatic disease..$^{35}$ Accordingly, an improvement of the tumor-selective action of TRAIL appears mandatory. Along this line, we and others have already shown earlier that tumor-targeting approaches through the generation of TNF ligand fusion proteins, which aim to restrict TNF ligand's full cytotoxic activity to the cancerous tissue are very promising approaches to improve therapeutic efficacy of TRAIL and other members of the TNF ligand family. ${ }^{4}$ Conventional fusion proteins of TRAIL and antibody-derived scFv fragments reveal homotrimeric proteins with a molecular mass of $\sim 150 \mathrm{kDa}$. This nearly corresponds in size to complete antibodies that are reported to penetrate tumor tissues slowly and nonuniformly. ${ }^{36}$ 

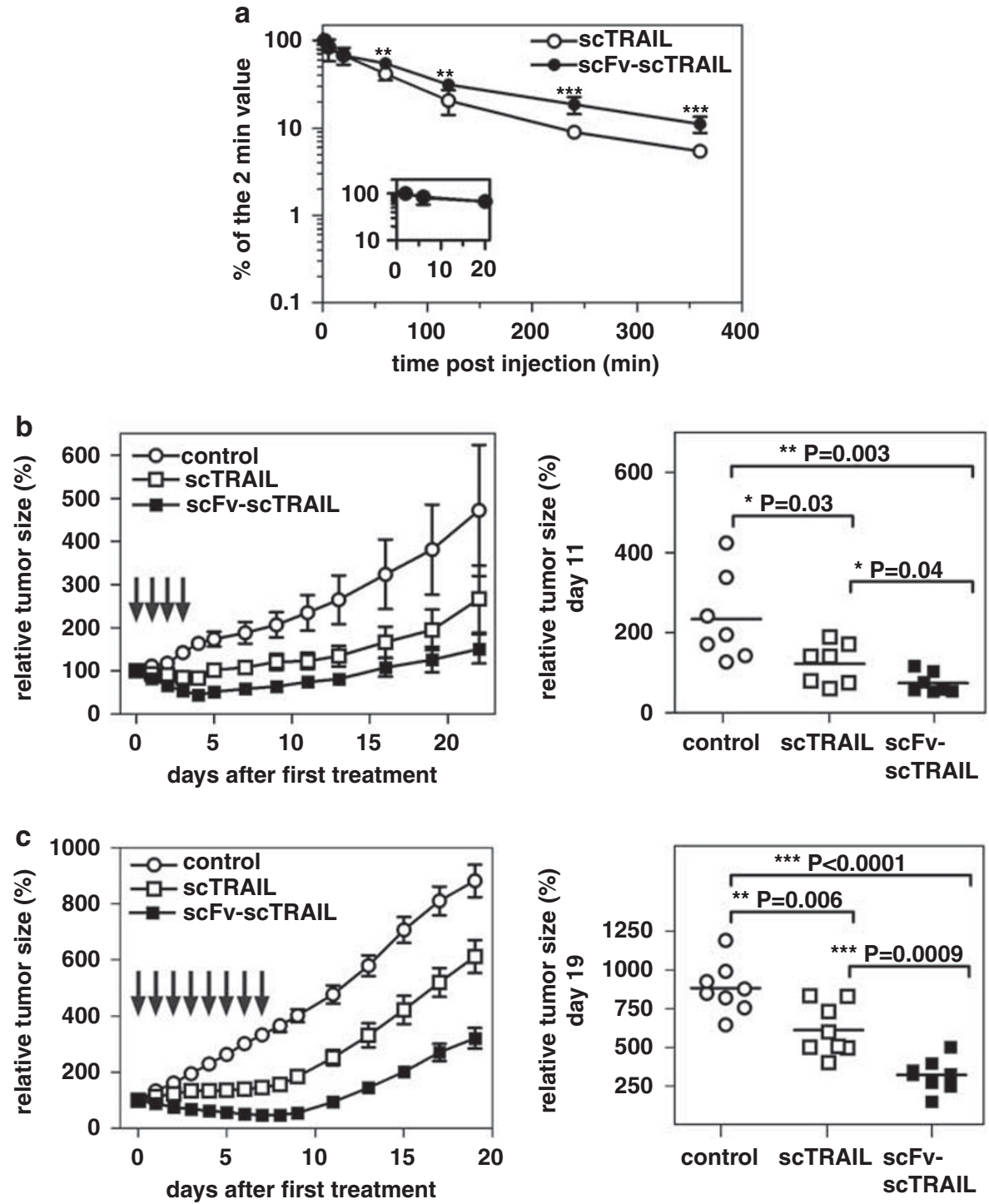

Figure 5 Pharmacokinetic properties and antitumoral activity of ScTRAIL and ScFv-scTRAIL. (a) Reduced elimination of scFv-scTRAIL from circulation. scTRAIL and ScFv-scTRAIL were detected by ELISA in serum samples obtained at the indicated time points after i.v. injection into Balb/c mice. For each protein three mice were treated. Significant differences between both proteins were marked by asterisks. (b) Antitumoral activity in a xenograft tumor model - local treatment. NMRI nu/nu mice were injected s.c. with $3 \times 10^{6}$ human colon carcinoma Colo205 cells. Treatment started when tumors reached about $25 \mathrm{~mm}^{2}$ ( 8 days after inoculation). Mice received four daily s.c. injections of $1 \mathrm{nmol}$ of the corresponding proteins beginning day $0 . n=7$ tumors per treatment group; symbols, mean of tumor volumes; bars, S.E.M. Individual tumor sizes at day 11 with bars indicating the mean are shown on the right. (c) Antitumoral activity in a xenograft tumor model - systemic treatment. $3 \times 10^{6}$ Colo205 cells were s.c. inoculated at the left and right dorsal site of NMRI nu/nu mice. Treatment started when tumors reached about $25 \mathrm{~mm}^{2}$ ( 5 days after inoculation). Mice received eight daily i.v. injections of $1 \mathrm{nmol}$ of the corresponding proteins beginning at day 0. $n=8$ tumors per treatment group; symbols, mean of tumor volumes; bars, S.E.M. Individual tumor sizes at day 19 with bars indicating the mean are shown on the right

Furthermore, antibody fragments may have an intrinsic tendency to aggregate, thereby potentially leading to higher order complexes, which in turn not only results in a further increase in size but may also cause target-independent TRAIL-R2 activity, too. To circumvent or at least minimize these potential problems, we have developed a new format of a TRAIL fusion protein based on ScTRAIL, a single polypeptide chain composed of three TRAIL monomers. Gel filtration and western blot analysis revealed that the ErbB2specific ScFv-scTRAIL fusion protein could be expressed in the expected monomeric state corresponding formally to a trimeric TRAIL variant. This is of great importance, as it became evident that the formation of high order complexes and aggregates of recombinant forms of TRAIL (e.g., His-TRAIL, crosslinked FLAG-TRAIL) increases the toxicity of the ligand to normal cells (reviewed by Koschny et al. ${ }^{16}$ ). As expected, the ErbB2-specific scFv-scTRAIL specifically bound to ErbB2-positive cells. This could be confirmed by the use of TRAILR-Fc fusion proteins to detect the ErbB2 bound SCFv-scTRAIL and by blocking of fusion protein binding with the ErbB2-specific antibody FRP5, thereby indicating predominant binding of the fusion protein by the antibody 
moiety to ErbB2 rather than by the TRAIL domain and TRAIL-R. Compared with the non-targeted ScTRAIL molecule, the tumor antigen-targeted scTRAIL fusion protein showed higher apoptotic activity in vitro on ErbB2-positive cells. This target antigen-dependent cytotoxicity could be confirmed by preincubation with competing ErbB2-specific antibody FRP5 or with ErbB2 peptide, restoring cell viability to a significant extent. We and others have previously described that, compared with soluble homotrimeric TRAIL, homotrimeric ScFv-TRAIL fusion proteins gain a higher activity on TRAIL-R2 upon cell surface binding by the targeting domain within the fusion proteins. ${ }^{13,26,27}$ Of note, although there is some controversial discussion on this, it has been shown that efficient activation of TRAIL-R2 is only reached by natural membrane TRAIL, crosslinked soluble or cell surface immobilized TRAIL. ${ }^{13,26,27,37,38}$ Importantly, the ScFv-scTRAIL fusion protein likewise converted into a functional equivalent of membrane TRAIL on target-antigen binding, and thus efficiently induced cell death already at low concentrations by TRAIL-R2, the predominant TRAILinduced apoptosis pathway in the cell lines studied here. Accordingly, receptor binding and activation could be blocked by TRAIL-R2-neutralizing monovalent Fab fragments. Moreover, Jurkat cells, which only express TRAIL-R2, but not ErbB2 and TRAIL-R1 are resistant against soluble scFvScTRAIL, whereas crosslinking of the fusion protein led to an increase in activity. These properties are especially important based on observations that on tumor cells TRAIL-R2 is more frequently and strongly expressed than TRAIL-R $1,{ }^{39}$ that apoptotic signaling through TRAIL-R2 may be more potent than through TRAIL-R $1^{14}$ and that, on the other hand, TRAILR2 may be a key regulator of cholestatic liver injury by induction of apoptosis in cholangiocytes. ${ }^{35}$ Consequently, the scFv-scTRAIL might be an appropriate therapeutic, which meets the criteria of high antitumor and low off-target activity. In vivo analyses in mice revealed that, on i.v. injection, the ScFv-scTRAIL and the ScTRAIL displayed biphasic pharmacokinetics. In the first phase, the calculated $\mathrm{t}_{\frac{1}{2}} \alpha$ of $\sim 35 \mathrm{~min}$ was similar for both proteins. In the second phase, the scFvscTRAIL displayed a slightly increased half-life with $\mathrm{t}_{\frac{1}{2}} \beta$ of $\sim 160 \mathrm{~min}$ compared with $125 \mathrm{~min}$ for the scTRAIL. This strongly contrasts to some of the published data for homotrimeric TRAIL, indicating a half-life of $3-5 \mathrm{~min}\left(\mathrm{t}_{\frac{1}{2}} \alpha\right)$ and $20 \mathrm{~min}\left(\mathrm{t}_{\frac{1}{2}} \beta\right)$ in rodents. ${ }^{40,41}$ Our results rather match with pharmacokinetic data of LZ-TRAIL, a trimer-stabilized TRAIL variant $\left(\mathrm{t}_{\frac{1}{2}} \alpha: 1.3 \mathrm{~h} ; \mathrm{t}_{\frac{1}{2}} \beta: 4.8 \mathrm{~h}^{8}\right)$. Most important, both singlechain TRAIL variants significantly reduced growth of established Colo205 tumors already at rather moderate doses, either on local or systemic treatment. In accordance with the in vitro bioactivity, the ScFv-scTRAIL displayed superior tumoricidal effects in vivo compared with the ScTRAIL. We propose that increased antitumoral activity is largely related to ErbB2-mediated tumor enrichment and the targeting-dependent increase in bioactivity rather than the slightly increased bioavailability. The observed re-growth of tumors upon cessation of treatment has also been reported with homotrimeric TRAIL and might overcome when the combination treatments with appropriate chemotherapeutics are conducted. ${ }^{9,10,40}$ Furthermore, we can exclude an effect of the ErbB2-specific antibody fragment scFvFRP5 on tumor growth, as addition of TRAIL-neutralizing antibodies completely restored cell viability on incubation with ScFv-scTRAIL. This is in accordance with previous reports showing that the mAb FRP5 did not affect tumor growth in vivo, despite even partial agonistic activity in vitro, evident from an increase in ErbB2 phosphotyrosine level and accelerated receptor turnover by antibody-mediated receptor crosslinking. ${ }^{42}$ In contrast, a monovalent ScFvFRP5-ETA fusion protein did not induce ErbB2-tyrosine phosphorylation, indicating that the activity of a monovalent ScFv fusion protein might be limited by the intrinsic turnover rate of its target receptor. ${ }^{43}$ We have own preliminary data confirming an increase in ErbB2phosphorylation on treatment of Colo205 cells with a conventional, homotrimeric ScFvFRP5-TRAIL fusion protein, whereas treatment with the monovalent ScFv-scTRAIL had no receptor-activating effect (data not shown). Accordingly, the single-chain design concept might be of advantage compared with a conventional fusion protein if target-antigen multimerization has to be avoided. Moreover, Stagg et al. $^{44}$ could show that combination of ErbB2 blockade and TRAIL-R2 activation can elicit potent synergistic antitumor activity. Thus, modifying the targeting module using an ErB2specific scFv antibody fragment also capable of inhibiting ErbB2 signaling might result in further increased ErbB2specific antitumor activity of an ScFv-TRAIL fusion protein. The here described antitumoral activity of the ScFv-scTRAIL fusion protein, which resulted in clear reduction in tumor size under treatment, appears superior to that of published preclinical data with homotrimeric TRAIL, regarding both the applied dose and the treatment periods. $7,8,40$ However, detailed side-by-side comparison of different TRAIL reagents are required to confirm this observation.

In conclusion, our data provide evidence that bioactive single-chain TRAIL fusion proteins can be generated. Furthermore, fusion to a target-specific scFv antibody results in target-dependent increase in TRAIL-mediated cytotoxicity in vitro, likely due to efficient TRAIL-R2 activation, and improves antitumoral activity in vivo. Accordingly, this design concept represents a promising strategy to enhance TRAIL's antitumoral action and to minimize potential unwanted off-target actions on normal tissues.

\section{Materials and Methods}

Plasmids and cell lines. The pIRESpuro-scTRAIL expression construct for human scTRAIL (aa 95-281) was generated by insertion of linker sequences, encoding four repetitions of the GGGS motif, between the three TRAIL modules as described previously for the construction of scTNF. ${ }^{20}$

The single-chain antigen binding protein scFvFRP5 for the ErbB2-specific pIRESpuro-scFv-scTRAIL expression construct was described previously. ${ }^{21}$ HEK293, Jurkat, Colo205, SKW6.4 and L929 cells were obtained from the American Type Culture Collection (Manassas, VA, USA) or DSMZ - Deutsche Sammlung von Mikroorganismen und Zellkulturen GmbH (Braunschweig, Germany). The HT1080 cells were a kind gift of W Rettig (Boehringer Ingelheim Pharma, Vienna, Austria). Cells were cultured in RPMI 1640 medium (Invitrogen, Karlsruhe, Germany) supplemented with $5 \%$ or $10 \%$ (SKW6.4) fetal calf serum (FCS, PAA Laboratories, Pasching, Austria).

Production and purification of recombinant proteins. The TRAIL fusion proteins were produced in HEK293 cells after stable transfection of the corresponding expression plasmids using Lipofectamine 2000 (Invitrogen) and selection of stable clones by limiting dilution. For protein production, stable clones were expanded and grown in RPMl $1640,5 \%$ FCS, to $90 \%$ confluency and 
subsequently cultured in serum-free Optimem (Invitrogen) supplemented with $50 \mu \mathrm{M} \mathrm{ZnCl}_{2}$, replacing media two times every 3 days. Supernatants were pooled and recombinant proteins were purified by affinity chromatography using anti-FLAG mAb M2 agarose (Sigma-Aldrich, Steinheim, Germany). The bound proteins were eluted with $100 \mu \mathrm{g} / \mathrm{ml}$ FLAG peptide (Sigma-Aldrich) and dialyzed against PBS. After concentrating purified proteins by dialysis on PEG35000, they were analyzed and quantified by SDS-PAGE, silver gel (Sigma-Aldrich) and gel filtration and stored at $4^{\circ} \mathrm{C}$.

Gel filtration. Protein samples were applied to a BioSuite250 HR SEC $(300 \times 7.8)$ column (Waters, Millipore Corp., Milford, MA, USA) equilibrated in PBS and eluted at a flow rate of $0.5 \mathrm{ml} / \mathrm{min}$.

Flow cytometry. Cells $\left(5 \times 10^{5}\right)$ were incubated for $2 \mathrm{~h}$ at $4^{\circ} \mathrm{C}$ with the indicated concentrations of the TRAIL fusion proteins. After washing the cells three times with PBS, $2 \% \mathrm{FCS}, 0.02 \%$ sodium azide, bound fusion proteins were detected by anti-human TRAlL mAb (MAB687) $(1 \mu \mathrm{g} / \mathrm{ml}$, R\&D Systems, Wiesbaden, Germany) and fluorescein isothiocyanate-labeled rabbit anti-mouse $\lg G A b$ $(1 \mu \mathrm{g} / \mathrm{ml}$, Sigma-Aldrich). ErbB2 expression was detected by anti-c-neu (AB-5) $(1 \mu \mathrm{g} / \mathrm{ml}$, Merck KGaA, Darmstadt, Germany) and fluorescein isothiocyanatelabeled rabbit anti-mouse lgG Ab (1 $\mu \mathrm{g} / \mathrm{ml}$, Sigma-Aldrich). Alternatively, cell-bound scFv-scTRAIL was detected by TRAILR-Fc fusion proteins $(5 \mu \mathrm{g} / \mathrm{ml}$ TRAILR1-Fc and $3 \mu \mathrm{g} / \mathrm{ml}$ TRAILR2-Fc) and fluorescein isothiocyanate-labeled anti-human IgG, Fc-specific antibodies (Sigma-Aldrich). For competition studies, FRP5 hybridoma supernatant or mouse lgG1 (Sigma-Aldrich) was added $30 \mathrm{~min}$ before addition of the SCFv-scTRAIL.

Cell death assays. HT1080 cells $\left(2 \times 10^{4} /\right.$ well), Colo205 cells $\left(5 \times 10^{4} /\right.$ well), SKW6.4 cells $\left(2 \times 10^{4} /\right.$ well) or Jurkat cells $\left(1 \times 10^{5} /\right.$ well) were cultured in $100 \mu \mathrm{l}$ culture medium in 96 -well plates. The next day, cells were treated in duplicates or triplicates with the indicated concentrations of the TRAIL fusion proteins or 'Killer-TRAIL' (Axxora Deutschland GmbH, Lörrach, Germany). After over night incubation, cell viability was determined either by crystal violet staining (HT1080) or the MTT method (Jurkat, Colo205, SKW6.4), ${ }^{45}$ except that for MTT staining a lysis buffer consisting of $15 \%$ SDS in DMF/dH $\mathrm{d}_{2} \mathrm{O}$ (1:1), $\mathrm{pH} 4.5$ (with $80 \%$ acetic acid) was used. To show target antigen- and TRAIL-R2-dependent induction of cell death, cells were preincubated for $30 \mathrm{~min}$ with FRP5 hybridoma supernatant ${ }^{46}$ $(\sim 10 \mu \mathrm{g} / \mathrm{ml})$ or a competing ErbB2 peptide $(10 \mu \mathrm{g} / \mathrm{ml})$ encompassing the FRP5-binding epitope ${ }^{21}$ or a neutralizing TRAIL-R2-specific Fab preparation $(50 \mu \mathrm{g} / \mathrm{ml})$, respectively. For inhibition of TRAIL bioactivity, anti-TRAIL antibody 2E5 $(1 \mu \mathrm{g} / \mathrm{ml}$, Axxora Deutschland $\mathrm{GmbH})$ was used. For co-culture assays, $1 \times 10^{4}$ Colo205 and $1 \times 10^{4} \mathrm{~L}$ L29 cells/well were seeded in 96-well plates. The next day, cells were challenged in duplicates with scTRAIL and scFv-scTRAIL, respectively. After additional $16 \mathrm{~h}$, cell viability was determined using the MTT method. Cell death assays with HT1080 and Colo205 cells were performed in the presence of $2.5 \mu \mathrm{g} / \mathrm{m}$ cycloheximide (Sigma-Aldrich) to sensitize cells for the induction of cell death

Pharmacokinetics. Animal care and all experiments performed were in accordance with federal guidelines and have been approved by the University and State authorities. Balb/c mice (female, 7 weeks, weight between 18 and $20 \mathrm{~g}$ 3 mice/group) received an i.v. injection of $400 \mathrm{pmol}$ of the recombinant proteins in a total volume of $100 \mu \mathrm{l}$. In time intervals of 2, 6, 20,60, 120, 240, $360 \mathrm{~min}$ and $24 \mathrm{~h}$, blood samples $(\sim 100 \mu \mathrm{l})$ were taken from the tail and incubated on ice. Clotted blood was centrifuged at $10000 \mathrm{~g}$ for $10 \mathrm{~min}, 4^{\circ} \mathrm{C}$, and serum samples were stored at $-80^{\circ} \mathrm{C}$. Serum concentrations of recombinant proteins were finally determined by ELISA (BD Biosciences, Heidelberg, Germany). For calculation, relative values of serum concentrations were analyzed with the first value $(2 \mathrm{~min})$ being set to $100 \%$. GraphPad Prism Software was used for curve fitting by nonlinear regression. The pharmacokinetic parameters AUC, $t_{\frac{1}{2}} \alpha$ and $t_{\frac{1}{2}} \beta$ were calculated by Excel using the first 3 times points to calculate $t_{\frac{1}{2}} \alpha$ and the last three time points to calculate $t_{\frac{1}{2}} \beta$. Statistics were determined by the unpaired $t$-test.

\section{Xenograft mouse models}

Local treatment. Six-week-old female NMRI nu/nu mice (Janvier Laboratories, Le Genest-Saint-Isle, France) were injected s.c. with $3 \times 10^{6}$ Colo205 cells in $100 \mu$ PBS. Treatment started when tumors reached $\sim 25 \mathrm{~mm}^{2}$. Mice received 4 daily s.c. injections of $1 \mathrm{nmol}$ of the various TRAIL fusion proteins in concentrated cell-culture supernatant. Supernatants were concentrated by dialysis on PEG35000.
The control group received $100 \mu \mathrm{l}$ concentrated cell-culture supernatant from empty vector-transfected cells at the same time intervals.

Systemic treatment. Colo205 cells $\left(3 \times 10^{6}\right)$ in $100 \mu$ l PBS were s.c. inoculated at the left and right dorsal sides of 6-week-old female NMRI nu/nu mice. Treatment started 5 days after tumor cell inoculation when tumors reached $\sim 25 \mathrm{~mm}^{2}$. Mice received 8 daily i.v. injections of $1 \mathrm{nmol}$ of the affinity purified TRAIL fusion proteins. The control group received $100 \mu \mathrm{l}$ PBS at the same time intervals. Tumor growth was monitored as described. ${ }^{47}$

\section{Conflict of interest}

The authors declare no conflict of interest.

Acknowledgements. We gratefully acknowledge technical support in the initial expression of scTRAIL by Sylvia Willy. Our work is supported by grants from Deutsche Krebshilfe, Grant no. 107551 and 108268 to HW and KP, from BMBF (Biochance plus, FKz 0315164) to KP and by a career fellowship award by the Peter and Traudl Engelhorn Foundation to JG.

1. Gerber DE. Targeted therapies: a new generation of cancer treatments. Am Fam Physician 2008; 77: 311-319.

2. Lejeune FJ, Lienard D, Matter M, Ruegg $C$. Efficiency of recombinant human TNF in human cancer therapy. Cancer Immun 2006; 6: 6 .

3. Call JA, Eckhardt SG, Camidge DR. Targeted manipulation of apoptosis in cancer treatment. Lancet Oncol 2008; 9: 1002-1011.

4. Gerspach J, Wajant H, Pfizenmaier K. Death ligands designed to kill: development and application of targeted cancer therapeutics based on proapoptotic TNF family ligands. Results Probl Cell Differ 2009; 49: 241-273.

5. Newsom-Davis T, Prieske S, Walczak H. Is TRAlL the holy grail of cancer therapy? Apoptosis 2009; 14: 607-623.

6. Wajant H. CD95L/FasL and TRAIL in tumour surveillance and cancer therapy. Cancer Treat Res 2006; 130: 141-165.

7. Ashkenazi A, Pai RC, Fong S, Leung S, Lawrence DA, Marsters SA et al. Safety and antitumor activity of recombinant soluble Apo2 ligand. J Clin Invest 1999; 104: 155-162.

8. Walczak H, Miller RE, Ariail K, Gliniak B, Griffith TS, Kubin M et al. Tumoricidal activity of tumor necrosis factor-related apoptosis-inducing ligand in vivo. Nat Med 1999; 5: 157-163.

9. Naka T, Sugamura K, Hylander BL, Widmer MB, Rustum YM, Repasky EA. Effects of tumor necrosis factor-related apoptosis-inducing ligand alone and in combination with chemotherapeutic agents on patients' colon tumors grown in SCID mice. Cancer Res 2002; 62: 5800-5806.

10. Hylander BL, Pitoniak R, Penetrante RB, Gibbs JF, Oktay D, Cheng J et al. The anti-tumor effect of Apo2L/TRAIL on patient pancreatic adenocarcinomas grown as xenografts in SCID mice. J Transl Med 2005; 3: 22.

11. Ashkenazi A, Herbst RS. To kill a tumor cell: the potential of proapoptotic receptor agonists. J Clin Invest 2008; 118: 1979-1990.

12. Falschlehner $\mathrm{C}$, Ganten TM, Koschny R, Schaefer U, Walczak H. TRAIL and other TRAIL receptor agonists as novel cancer therapeutics. Adv Exp Med Biol 2009; 647: 195-206.

13. Wajant $\mathrm{H}$, Moosmayer D, Wuest $\mathrm{T}$, Bartke $T$, Gerlach $\mathrm{E}$, Schonherr U et al. Differential activation of TRAIL-R1 and -2 by soluble and membrane TRAIL allows selective surface antigen-directed activation of TRAIL-R2 by a soluble TRAIL derivative. Oncogene 2001; 20: $4101-4106$

14. Kelley RF, Totpal K, Lindstrom SH, Mathieu M, Billeci K, DeForge L et al. Receptorselective mutants of apoptosis-inducing ligand 2/tumor necrosis factor-related apoptosisinducing ligand reveal a greater contribution of death receptor (DR) 5 than DR4 to apoptosis signaling. J Biol Chem 2005; 280: 2205-2212.

15. Johnstone RW, Frew AJ, Smyth MJ. The TRAIL apoptotic pathway in cancer onset, progression and therapy. Nat Rev Cancer 2008; 8: 782-798.

16. Koschny R, Walczak H, Ganten TM. The promise of TRAIL - potential and risks of a novel anticancer therapy. J Mol Med 2007; 85: 923-935.

17. Holbro T, Hynes NE. ErbB receptors: directing key signaling networks throughout life. Annu Rev Pharmacol Toxicol 2004; 44: 195-217.

18. Baselga J, Swain SM. Novel anticancer targets: revisiting ERBB2 and discovering ERBB3. Nat Rev Cancer 2009; 9: 463-475.

19. Hynes NE, Macdonald G. ErbB receptors and signaling pathways in cancer. Curr Opin Cell Biol 2009; 21: 177-184.

20. Krippner-Heidenreich A, Grunwald I, Zimmermann G, Kuhnle M, Gerspach J, Sterns T et al. Single-chain TNF, a TNF derivative with enhanced stability and antitumoral activity. J Immunol 2008; 180: 8176-8183.

21. Wels W, Harwerth IM, Zwickl M, Hardman N, Groner B, Hynes NE. Construction, bacterial expression and characterization of a bifunctional single-chain antibody-phosphatase fusion protein targeted to the human erbB-2 receptor. Biotechnology (NY) 1992; 10: 1128-1132. 
22. Hynes NE, Gerber HA, Saurer S, Groner B. Overexpression of the c-erbB-2 protein in human breast tumor cell lines. J Cell Biochem 1989; 39: 167-173.

23. Luciano F, Ricci JE, Herrant M, Bertolotto C, Mari B, Cousin JL et al. T and B leukemic cell lines exhibit different requirements for cell death: correlation between caspase activation, DFF40/DFF45 expression, DNA fragmentation and apoptosis in T cell lines but not in Burkitt's lymphoma. Leukemia 2002; 16: 700-707.

24. Guo F, Sigua C, Tao J, Bali P, George P, Li Y et al. Cotreatment with histone deacetylase inhibitor LAQ824 enhances Apo-2L/tumor necrosis factor-related apoptosis inducing ligand-induced death inducing signaling complex activity and apoptosis of human acute leukemia cells. Cancer Res 2004; 64: 2580-2589.

25. Muhlenbeck F, Schneider P, Bodmer JL, Schwenzer R, Hauser A, Schubert G et al. The tumor necrosis factor-related apoptosis-inducing ligand receptors TRAIL-R1 and TRAIL-R2 have distinct cross-linking requirements for initiation of apoptosis and are non-redundant in JNK activation. J Biol Chem 2000; 275: 32208-32213.

26. Bremer E, Kuijlen J, Samplonius D, Walczak H, de Leij L, Helfrich W. Target cell-restricted and -enhanced apoptosis induction by a scFv:sTRAIL fusion protein with specificity for the pancarcinoma-associated antigen EGP2. Int J Cancer 2004; 109: 281-290.

27. Bremer E, Samplonius DF, Peipp M, van Genne L, Kroesen BJ, Fey GH et al. Target cell-restricted apoptosis induction of acute leukemic $T$ cells by a recombinant tumor necrosis factor-related apoptosis-inducing ligand fusion protein with specificity for human CD7. Cancer Res 2005; 65: 3380-3388.

28. Ling J, Herbst RS, Mendelson DS, Eckhardt SG, O'Dwyer P, Ebbinghaus S et al. Apo2L/ TRAIL pharmacokinetics in a phase la trial in advanced cancer and lymphoma. J Clin Oncol 2006; $24: 3047$.

29. Herbst RS, Mendelson DS, Ebbinghaus S, Gordon MS, O'Dwyer P, Lieberman G et al. A phase I safety and pharmacokinetic (PK) study of recombinant Apo2L/TRAIL, an apoptosis-inducing protein in patients with advanced cancer. J Clin Oncol 2006; 24: 3013.

30. Ishimura N, Isomoto H, Bronk SF, Gores GJ. Trail induces cell migration and invasion in apoptosis-resistant cholangiocarcinoma cells. Am J Physiol Gastrointest Liver Physiol 2006; 290: G129-G136.

31. Ehrhardt H, Fulda S, Schmid I, Hiscott J, Debatin KM, Jeremias I. TRAIL induced survival and proliferation in cancer cells resistant towards TRAIL-induced apoptosis mediated by NF-kappaB. Oncogene 2003; 22: 3842-3852.

32. Volkmann X, Fischer U, Bahr MJ, Ott M, Lehner F, MacFarlane M et al. Increased hepatotoxicity of tumor necrosis factor-related apoptosis-inducing ligand in diseased human liver. Hepatology 2007; 46: 1498-1508.

33. Babu CK, Suwansrinon K, Bren GD, Badley AD, Rizza SA. HIV induces TRAIL sensitivity in hepatocytes. PLoS One 2009; 4: e4623.

34. Mundt B, Wirth T, Zender L, Waltemathe M, Trautwein C, Manns MP et al. Tumour necrosis factor related apoptosis inducing ligand (TRAIL) induces hepatic steatosis in viral hepatitis and after alcohol intake. Gut 2005; 54: 1590-1596.

35. Takeda K, Kojima Y, Ikejima K, Harada K, Yamashina S, Okumura K et al. Death receptor 5 mediated-apoptosis contributes to cholestatic liver disease. Proc Natl Acad Sci USA 2008; 105: $10895-10900$.
36. Holliger P, Hudson PJ. Engineered antibody fragments and the rise of single domains Nat Biotechnol 2005; 23: 1126-1136.

37. Berg D, Lehne M, Muller N, Siegmund D, Munkel S, Sebald W et al. Enforced covalent trimerization increases the activity of the TNF ligand family members TRAIL and CD95L. Cell Death Differ 2007; 14: 2021-2034.

38. Assohou-Luty C, Gerspach J, Siegmund D, Muller N, Huard B, Tiegs G et al. A CD40CD95L fusion protein interferes with CD40L-induced prosurvival signaling and allows membrane CD40L-restricted activation of CD95. J Mol Med 2006; 84: 785-797.

39. Ashkenazi A. Targeting death and decoy receptors of the tumour-necrosis factor superfamily. Nat Rev Cancer 2002; 2: 420-430.

40. Kelley SK, Harris LA, Xie D, DeForge L, Totpal K, Bussiere J et al. Preclinical studies to predict the disposition of Apo2L/tumor necrosis factor-related apoptosis-inducing ligand in humans: characterization of in vivo efficacy, pharmacokinetics, and safety. J Pharmacol Exp Ther 2001; 299: 31-38.

41. Xiang H, Nguyen CB, Kelley SK, Dybdal N, Escandon E. Tissue distribution, stability, and pharmacokinetics of Apo2 ligand/tumor necrosis factor-related apoptosis-inducing ligand in human colon carcinoma COLO205 tumor-bearing nude mice. Drug Metab Dispos 2004; 32: $1230-1238$

42. Harwerth IM, Wels W, Schlegel J, Muller M, Hynes NE. Monoclonal antibodies directed to the erbB-2 receptor inhibit in vivo tumour cell growth. Br J Cancer 1993; 68: $1140-1145$.

43. Wels W, Moritz D, Schmidt M, Jeschke M, Hynes NE, Groner B. Biotechnological and gene therapeutic strategies in cancer treatment. Gene 1995; 159: 73-80.

44. Stagg J, Sharkey J, Pommey S, Young R, Takeda K, Yagita H et al. Antibodies targeted to TRAIL receptor-2 and ErbB-2 synergize in vivo and induce an antitumor immune response. Proc Natl Acad Sci USA 2008; 105: 16254-16259.

45. Wuest T, Gerlach E, Banerjee D, Gerspach J, Moosmayer D, Pfizenmaier K TNF-Selectokine: a novel prodrug generated for tumor targeting and site-specific activation of tumor necrosis factor. Oncogene 2002; 21: 4257-4265.

46. Harwerth IM, Wels W, Marte BM, Hynes NE. Monoclonal antibodies against the extracellular domain of the erbB-2 receptor function as partial ligand agonists. J Biol Chem 1992; 267: 15160-15167.

47. Asher A, Mule JJ, Reichert CM, Shiloni E, Rosenberg SA. Studies on the anti-tumor efficacy of systemically administered recombinant tumor necrosis factor against several murine tumors in vivo. J Immunol 1987; 138: 963-974.

Cell Death and Disease is an open-access journal published by Nature Publishing Group. This work is licensed under the Creative Commons Attribution-Noncommercial-No Derivative Works 3.0 Unported License. To view a copy of this license, visit http://creativecommons.org/licenses/by-nc-nd/3.0/ 